\title{
Magnification of Fear and Intention of Avoidance of Non-Experienced Dental Treatment
}

Chia-Shu Lin ( $\nabla$ winzlin@ym.edu.tw )

National Yang Ming University

Chen-Yi Lee

Kaohsiung Medical University

Li-Ling Chen

National Yang Ming University

Tze-Fang Wang

National Yang Ming University

\section{Research Article}

Keywords: fear, avoidance, dental anxiety, pain

Posted Date: December 14th, 2020

DOl: https://doi.org/10.21203/rs.3.rs-119910/v1

License: (c) (1) This work is licensed under a Creative Commons Attribution 4.0 International License. Read Full License 


\section{Abstract}

Background. Dental fear is associated with the experience of prior dental treatment and avoidance of dental visits. It remains unclear if individuals show an intention of avoidance (IA) towards treatments that they have not received (i.e., non-experienced dental treatment).

Methods. We investigated fear/IA of 12 common conditions of dental treatment of 402 adults. If subjects have experienced the condition, fear and IA were assessed based on subjects' prior experience (i.e., ExpFear/ExpIA). If they have not experienced the condition, fear and IA were assessed based on their anticipation (i.e., NExpFear/NExpIA). Trait dental anxiety was assessed using the Index of Dental Anxiety and Fear (IDAF-4C+).

Results. We found that (A) NExpFear and NExpIA were significantly higher than ExpFear and ExpIA, respectively. (B) The IDAF-4C+ scores are positively correlated with NExpFear/NExpIA and negatively correlated with the magnification of fear (i.e., the discrepancy in the fear/IA of non-experienced vs. experienced conditions). (C) The condition 'extraction of a wisdom tooth' and 'root canal treatment' showed the highest ratings on NExpFear.

Conclusions. Individuals may develop a high degree of fear and IA of the treatment they have not received. Trait dental anxiety plays a key role in the magnification of the fear of non-experienced treatment.

\section{Introduction}

Traditional views hold that one's prior experience of dental treatment plays a key role in shaping dental fear/anxiety [1-3]. Clinical evidence supports the notion that fear of dental treatment is closely associated with previous negative treatment experience [4-7]. A recent cross-sectional study reported that the experience of past dental appointments may influence patients" intentions of future appointments, highlighting the importance of patients' evaluation and anticipation for their intentions of dental attendance [8]. Notably, patients' anticipation of fear and pain towards coming treatment may not reliably reflect their actual experience of treatment. Individuals may expect a stimulus to be more painful than what they actually perceived $[9,10]$. Moreover, individuals may 'overestimate' the fear of pain of the dental treatment that they who have not experienced, compared to those they have experienced in the past [11]. Such an 'overestimation' of fear of pain may be associated with trait dental anxiety [11].

Critically, fear and anxiety are not only associated with pain but also avoidance of dental treatment. Around $15 \%$ to $20 \%$ of the adult population avoid or delay visiting dentists [12-14], which results in a 'vicious cycle' of oral health [12]. While individuals may magnify their fear of the treatment that they have not received [11], it has remained unclear if individuals also show an intention of avoidance (IA) towards the treatment that they have not experienced (i.e., non-experienced treatment). The current study aims to investigate the association between fear/IA of experienced and non-experienced conditions of dental treatment in adults. 
Because dental treatment consists of a variety of procedures, which show different anxiety-stimulating effects [15], we investigated fear and IA of 12 conditions about common dental procedures. Self-ratings of fear and IA were assessed based on subjects' prior experience if they have experienced a condition (i.e., ExpFear/ExplA), or based on their anticipation if they have not experienced a condition (i.e., NExpFear/NExplA). Three major hypotheses were tested:

A) Hypothesis 1: Based on the previous findings of overestimation about fear of dental pain [11], we hypothesized that fear/IA ratings are higher for the non-experienced vs. the experienced conditions of treatment.

B) Hypothesis 2A: Due to the close relationship between fear and avoidance [13], we hypothesized that one's fear of experienced treatment is positively correlated with not only the IA of experienced treatment but also the IA of non-experienced treatment. Hypothesis 2B: Anxiety refers to a future-oriented state responding to an anticipated threat [16]. Therefore, we hypothesized that individual scores of trait dental anxiety are positively correlated with their fear/IA of non-experienced treatment.

C) Hypothesis 3: Different dental procedures have different anxiety-stimulating effects [15]. Some dental procedures may show a greater magnification factor (i.e., a greater discrepancy in the fear/IA of nonexperienced vs. experienced conditions). We hypothesized that individuals would magnify fear/IA to a lesser degree for the conditions that more people have experienced (i.e., with a higher prevalence).

\section{Materials \& Methods}

\section{Participants}

Two samples were recruited independent for the current study: 201 participants from a local community recruited via advertisement and 201 dental patients from the outpatient clinic of Taipei Veterans General Hospital (Table 1). The participants provided written informed consent, approved by the Institutional Review Board of National Yang-Ming University (ID: YM106095E) and Taipei Veterans General Hospital (ID: 2018-12-003AC) before all the assessment started.

\section{Experimental Procedure}

\section{Dental Treatment Experience}

The questionnaire for dental treatment experience was customized with descriptions about 12 conditions of common dental procedures (Table 2), based on our previous study [17] and an earlier study [11] that adopted a set of selected dental procedures. To ensure that the conditions are common to most patients, we excluded the procedures about complicated orofacial surgery or orthodontic therapy. Trait dental anxiety was assessed using the Chinese version [18] of the Index of Dental Anxiety and Fear (IDAF-4C+) [19]. 
For each condition of dental treatment, the participants were asked to indicate (a) whether they have experienced that condition of treatment in the past (i.e., history of treatment, HT), the degree of (b) fear of the condition (Fear), and (c) intention of avoidance of the condition (IA), respectively, according to the following instruction:

A. 'If you have previously experienced a condition about the treatment, please rate the degree of fear and intention of avoidance about that condition, by recalling your prior experience about it.'

B. 'If you have never experienced a condition about the treatment, please rate the degree of fear and intention of avoidance about that condition, by anticipating what you would feel about it, according to what you know the treatment.'

All the ratings were scored based on a 10-point numerical rating scale, ranging from 1 (the least degree of fear/IA) to 10 (the maximal degree of fear/IA). The scores of HT, fear of experienced treatment (ExpFear), IA of experienced treatment (ExpIA), fear of non-experienced treatment (NExpFear), and IA of nonexperienced treatment (NExpIA), were calculated by including the ratings from all the 12 conditions, according to the following methods:

a. The conditions that subjects have experienced were indexed by the value ' 1 ' and those they have not experienced were indexed by the value ' 0 '. The average HT was the mean of the 12 values, denoting the proportion of conditions that a subject has experienced.

b. ExpFear and ExpIA were calculated as the mean of Fear and IA, respectively, across the conditions that subjects have experienced (i.e., the conditions valued as ' 1 ' for $\mathrm{HT}$ ).

c. NExpFear and NExpIA were calculated as the mean of Fear and IA, respectively, across the conditions that subjects have not experienced (i.e., the conditions valued as ' 0 ' for HT).

\section{Statistical Analysis}

\section{Analysis of descriptive statistics}

The analysis of descriptive statistics was conducted for all the variables (age, sex, and the average of HT, IDAF-4C+, ExpFear, ExpIA, NExpFear, and NExpIA), across all subjects $(n=402)$ and respectively for the community and the clinical groups ( $n=201$ for each). Normality of the score distribution was assessed using the Shapiro-Wilk test, with $p<0.1$ indicating non-normality.

For each of the 12 conditions of treatment, the analysis descriptive statistics was also conducted for the following variables: IDAF-4C+, ExpFear, ExpIA, NExpFear, and NExpIA. For each condition, the prevalence of experiencing a condition was calculated as the mean HT averaged across all subjects. The magnification factor, i.e., the discrepancy in the fear/IA of non-experienced vs. experienced conditions, was calculated as follows:

(a) Magnification factor of fear $=100 \% \mathrm{X}(\mathrm{NExpFear}-$ ExpFear $) /$ ExpFear 
(b) Magnification factor of $I A=100 \% X(N E x p I A-$ ExpIA $) /$ ExpIA

\section{Analysis 1: Comparison between experienced and non-experienced fear/IA}

To test Hypothesis 1, we performed the Wilcoxon signed-rank test for comparing the scores between ExpFear and NExpFear as well as the scores between ExpIA and FearlA, across all subjects. The choice of non-parametric method is based on the non-normality of the distribution of the scores (Table 1).

\section{Analysis 2: Association between dental anxiety, experienced and non-experienced fear/IA}

To test Hypotheses 2A, we examined the strength of association between (a) ExpFear and ExpIA and (b) between ExpFear and NExpIA, using the Spearman's rho coefficient. To test Hypotheses 2B, we examined the strength of association between (a) IDAF-4C+ and NExpFear and (b) between IDAF-4C+ and NExpIA.

\section{Analysis 3: Association between the magnification factor of a dental condition and the prevalence of experiencing it}

To test Hypothesis 3, we examined the strength of association between (a) the prevalence of experiencing a condition and its magnification factor of fear and (b) the association between the prevalence and its magnification factor of IA, using the Spearman's rho coefficient.

All the statistical analyses were performed using IBM SPSS Statistics (v. 24). For all the statistical tests, the level of statistical significance (alpha) was 0.05 .

\section{Results}

\section{Descriptive Analysis}

The results of descriptive analyses were shown in Table 1. Due to the non-normality of the data distribution, we adopted non-parametric methods for the subsequent statistical analyses. The proportion of sex was not significantly different (two-tailed Chi-square test with the Yates continuity correction, $p$ > 0.05) and age, IDAF-4C + score, ExpFear, ExpIA, NExpFear, and NExpIA were not significantly different (two-tailed Mann-Whitney $U$ test, $p>0.05$ ) between the two groups.

Table 2 revealed that the conditions "root canal treatment" and "extraction of a wisdom tooth" showed the highest scores of both ExpFear and NExpFear. The conditions "extraction of primary tooth" and "failure to relieve postoperative pain" showed the highest scores of ExpIA. In contrast, the conditions "receiving local anesthesia" and "root canal treatment" showed the highest scores of NExpIA and also the highest magnification factor of IA (Table 2).

\section{Analysis 1: Comparison between experienced and non- experienced fear/IA}


Confirming Hypothesis 1, we found that NExpFear was significantly higher than ExpFear (two-tailed Wilcoxon signed-rank test, $\mathrm{p}<0.001$ ) and NExpIA was significantly higher than ExpIA (two-tailed Wilcoxon signed-rank test, $p<0.001)$ (Fig. 1A).

\section{Analysis 2: Association between dental anxiety, experienced and non-experienced fear/IA}

Confirming Hypothesis 2, we found a positive correlation between ExpFear and ExplA (rho $=0.62, p<$ 0.001 ) as well as a positive correlation between ExpFear and NExpIA (rho $=0.51, p<0.001$ ) (Fig. 1B). Additionally, we found a positive correlation between NExpFear and NExpIA ( $r h o=0.64, p<0.001$ ) as well as a positive correlation between ExpIA and NExpIA ( $r h o=0.67, p<0.001)$. Confirming Hypotheses $2 B$, we found a positive correlation between IDAF-4C + and NExpFear (rho $=0.53, p<0.001$ ) as well as a positive correlation between IDAF-4C + and NExpIA (rho = 0.49, p < 0.001) (Fig. 1C). Additionally, IDAF-4C + was positively correlated with ExpFear (rho $=0.63, p<0.001)$ and $\operatorname{ExplA}($ rho $=0.59, p<0.001)$.

We additionally examined if the discrepancy between non-experienced (NExpFear) and experienced (ExpFear) ratings, i.e., the magnification factor of fear, was associated with dental anxiety. The analysis showed a significant negative correlation between the magnification factor of fear and IDAF-4C+ (rho=-0.14, $p=0.008$ ) (Fig. 1D). The correlation between the magnification factor of IA and IDAF-4C + did not show a significant result (rho $=0.05, p=0.3$ ) (Fig. 1D).

Analysis 3: Association between the magnification factor of a dental condition and the prevalence of experiencing it

We first identified the association between ExpFear and NExpFear as well as the association between ExpIA and NExpIA, across the 12 conditions. As shown in Fig. 2A, the scores of experienced and nonexperienced conditions showed a significant positive correlation for fear (rho $=0.85, p<0.001$ ) but not for IA (rho $=0.54, p=0.07)$. Critically, we did not find a significant correlation between the prevalence and the magnification factor of fear ( $r$ o $=0, p=1.0$ ) or between the prevalence and the magnification factor of IA ( $r$ ho $=0.29, p=0.37$ ). The results thus disconfirmed Hypothesis 3 . However, an additional analysis revealed that the prevalence is only negatively correlated with ExplA ( $r h o=-0.63, p=0.027$ ) (Fig. 2B). The correlation between the prevalence and ExpFear, NExpFear, or NExpIA, was not statistically significant.

\section{Discussion}

\section{Major findings from the current study}

The current study aims to investigate the association between fear/IA of experienced and nonexperienced conditions of dental treatment in adults. Our major findings are:

A. NExpFear was significantly higher than ExpFear. Likewise, NExpIA was significantly higher than ExplA (Fig. 1A). 
B. ExpFear is positively correlated with ExplA as well as NExpIA (Fig. 1B). The individual IDAD-4C+ scores are positively correlated with NExpFear and NExpIA (Fig. 1C). Moreover, they are negatively correlated with the magnification factor of fear (Fig. 1D).

C. Among conditions of dental treatment, 'extraction of a wisdom tooth' and 'root canal treatment' showed the highest ratings on ExpFear and NExpFear (Table 2). The prevalence of experiencing a condition is negatively correlated with ExpIA (Fig. 2B)).

\section{Association between fear and intention of avoidance}

It has been widely observed that prior experience of receiving dental procedures is associated with the fear of dental treatment [4-7]. For example, patients with negative experience about dental injections would report stronger pain and anxiety during treatment [10]. Fear and pain are also associated with patients' memory about receiving a procedure [20]. Notably, fear is considered a critical factor of avoidance of dental treatment $[12-14,21]$. Our findings revealed that fear may be associated with the intention of avoidance of dental treatment, even if individuals have not experienced the treatment. The findings implied that apart from prior experience, other factors may play a key role in avoidance. Apart from personal experience, social learning via language communication or observation also contributed to the development of fear [22]. Furthermore, pain can be potentiated by imagination [23] or the iconic impressions described by mass media [24].

Personal factors, such as trait dental anxiety, may play a key role in fear/IA of non-experienced conditions of treatment. Increased anxiety is associated with the response towards a future-oriented and anticipated threat [16], consistent with the condition when patients receive a procedure they have never met before. Critically, we found that an increased trait dental anxiety, indexed by the IDAF-4C + score, was associated with a decreased magnification factor of fear (Fig. 1D). The finding echoed the conclusion from van Wijk and Hoogstraten's work, which reported that the 'overestimation' of fear of pain was reduced in the sample of highly anxious patients, relative to the non-anxious group [11]. By extending this finding, we found that on the individual basis, subjects with a higher trait dental anxiety tended to rate the fear of the procedures not experienced higher than the fear of the procedures they have experienced (Fig. 1D). Our findings highlight that trait dental anxiety not only reflects increased pain [25] but also signifies a greater risk for patients to avoid the procedures even they have never experienced.

\section{Fear of dental treatment of common procedures}

Our findings revealed that the experience of more invasive procedures (e.g., root canal treatment and extraction of a wisdom tooth) were fearful for both the subjects who have and have not experienced it (Table 2 and Fig. 2A). Consistently, the study by van Wijk and Hoogstraten has reported that surgical procedures (e.g., "Having a lump cut open in the mouth", "Being drilled in the jawbone", and "An incision in the gums", tooth extraction, and root canal treatment, as the conditions with a high degree of overestimation in fear of pain [11]. Our results also echoed the findings from Oosterink et al., which revealed that root canal treatment and extraction of a molar as the dental experiences with the highest anxiety-provoking score (\#3 and \#5 in the ranking, respectively) among 67 dental stimuli [15]. 
By extending these findings, we identified a positive correlation between ExpFear and NExpFear, across the conditions (Fig. 2A). The findings may be interpreted from two aspects. First, the fear perceived by the patients who experienced a treatment (i.e., ExpFear) may be disseminated to people who have not experienced it, probably, via media or social networks, because fear can be acquired by social learning [22]. This interpretation is consistent with the findings that these two conditions are not rare to the subjects (with a prevalence of $68 \%$ and $38 \%$, respectively) (Table 2). A second interpretation is that a negative impression (e.g., "Tooth extraction must be scaring!" has already existed, and therefore, people have already expected a greater fear towards the treatment (i.e., a greater NExpFear) and the patients who experienced it just re-confirmed that impression (i.e., a greater ExpFear). The hypotheses regarding the cause-effect relationship between ExpFear and NExpFear may require further investigation.

\section{Intention of avoidance of dental treatment of common procedures}

Do the fearful experience of dental treatment make people avoid receiving further dental treatment? Our results have shown some critical clues regarding the fear-avoidance association. First, ExpFear was positively correlated with NExpIA (Fig. 1B), suggesting that prior experience may play a key role in the IA of the non-experienced treatment. Second, the IA scores are generally lower than the fear scores. As shown in Table 1, the median for ExpIA and NExpIA is 1.2 and 1.8, respectively, but the median for ExpFear and NExpFear is 3.1 and 4.3, respectively. The findings suggested that even though stronger fear is associated with a higher IA, in general, people are likely to attend dental treatment, evidenced by the relatively lower IA scores. In terms of the Health Belief Model [26], patients may see dentists because they perceive the symptom more severe (e.g., symptomatic pulpitis), even with a greater fear about the treatment (e.g., receiving root canal treatment). Also, the low IA may be associated with a decreased barrier of dental attendance. The approachability and availability to see a dentist in Taiwan (especially in the urban area) [27] and the lower financial burden of dental treatment due to National Health Insurance [28] may contribute to the lower IA of dental treatment.

\section{Limitations of the study}

The results from our research need to be interpreted with several considerations from the study design. First, the dental experience questionnaire that we adopted only focuses on 12 conditions related to common dental procedures. Therefore, the findings cannot be generalized to the treatment associated with complicated or elective procedures, such as orthodontic, prosthodontic, or cosmetic therapies. Second, due to the non-normal distribution of scores, we do not perform more sophisticated analyses (e.g., multivariable analyses) for investigating the relationship between each variable. Third, it is noteworthy that patients' intention of attendance of dental visits is associated with positive expectations, such as getting pain relieved or improving mastication. The interplay between the negative (fear) and positive expectations would need further investigation.

\section{Clinical implications}


Based on our novel findings presented here, we suggest three aspects for further considerations in clinical practice.

A. Individuals with a higher trait dental anxiety may tend to magnify the fear of the procedures that they have not experienced. Therefore, the assessment of dental anxiety will be helpful for predicting patients' dental-visiting experience.

B. Extraction of a wisdom tooth and root canal treat present the highest fear regardless of individual prior experience. Therefore, dentists may pay attention if a negative impression about the treatment has been cast on patients, leading to their fear and avoidance.

C. Finally, recent evidence has gradually disclosed the biological mechanisms of dental fear, including its association with genetic variations [29] and brain activation [17, 30]. The biological mechanisms underlying fear/avoidance towards non-experienced stimuli would require further investigation.

\section{Conclusion}

Our novel findings suggest that individuals may develop a high degree of fear and intention to avoidance toward the conditions of dental treatment they have not experienced. Individual variations in trait dental anxiety play a key role in the magnification of the fear of non-experienced treatment.

\section{Declarations}

\section{Ethics approval and consent to participate}

The study was approved by the Institutional Review Board of National Yang-Ming University (ID: YM106095E) and Taipei Veterans General Hospital (ID: 2018-12-003AC). The participants provided written informed consent before all the assessment started, in accordance with the Declaration of Helsinki.

\section{Consent for publication}

Not applicable.

\section{Availability of data and materials}

The datasets generated during and analyzed during the current study are not publicly available due to regulations on the privacy of the subjects according to the guidelines from local Internal Review Board but are available from the corresponding author on reasonable request.

\section{Competing interests}

Conflict of Interest: All the authors declare no conflict of interest.

\section{Funding}


C-S. Lin was funded by the Ministry of Science and Technology of Taiwan (MOST 107-2314-B-010-030MY2).

\section{Authors' contributions}

CS Lin, CY Lee, and TF Wang conceptualized and designed the research. CS Lin, CY Lee, and TF Wang conducted the research. CS Lin and LL Chen collected data. CS Lin and LL Chen analyzed data. CS Lin drafted the manuscript. CS Lin, CY Lee, and TF Wang finalized and approved the manuscript.

\section{Acknowledgements}

Not applicable.

\section{References}

1. Milgrom P, Weinstein P, Getz T: Treating fearful dental patients. Seattle, U.S.A.: University of Washington Continuing Dental Education; 1994.

2. Ost L-G, Skaret E: Cognitive behavioral therapy for dental phobia and anxiety. Chichester, U.K.: John Wiley \& Sons; 2013.

3. Weiner AA: The Fearful Dental Patients: A Guide to Understanding and Managing. Ames, lowa, U.S.A.: Wiley-Blackwell; 2011.

4. Locker D, Liddell A, Dempster L, Shapiro D: Age of onset of dental anxiety. Journal of dental research 1999, 78(3):790-796.

5. Locker D, Thomson WM, Poulton R: Psychological disorder, conditioning experiences, and the onset of dental anxiety in early adulthood. Journal of dental research 2001, 80(6):1588-1592.

6. Maggirias J, Locker D: Psychological factors and perceptions of pain associated with dental treatment. Community dentistry and oral epidemiology 2002, 30(2):151-159.

7. Berggren U, Meynert G: Dental fear and avoidance: causes, symptoms, and consequences. J Am Dent Assoc 1984, 109(2):247-251.

8. Schneider A, Andrade J, Tanja-Dijkstra K, White M, Moles DR: The psychological cycle behind dental appointment attendance: a cross-sectional study of experiences, anticipations, and behavioral intentions. Community dentistry and oral epidemiology 2016, 44(4):364-370.

9. Klages U, Ulusoy O, Kianifard S, Wehrbein H: Dental trait anxiety and pain sensitivity as predictors of expected and experienced pain in stressful dental procedures. European journal of oral sciences 2004, 112(6):477-483.

10. van Wijk A, Lindeboom JA, de Jongh A, Tuk JG, Hoogstraten J: Pain related to mandibular block injections and its relationship with anxiety and previous experiences with dental anesthetics. Oral surgery, oral medicine, oral pathology and oral radiology 2012, 114(5 Suppl):S114-119.

11. van Wijk AJ, Hoogstraten J: Experience with dental pain and fear of dental pain. Journal of dental research 2005, 84(10):947-950. 
12. Armfield JM: Predicting dental avoidance among dentally fearful Australian adults. European journal of oral sciences 2013, 121(3 Pt 2):240-246.

13. Armfield JM: What goes around comes around: revisiting the hypothesized vicious cycle of dental fear and avoidance. Community dentistry and oral epidemiology 2013, 41(3):279-287.

14. Nicolas E, Collado V, Faulks D, Bullier B, Hennequin M: A national cross-sectional survey of dental anxiety in the French adult population. BMC oral health 2007, 7:12.

15. Oosterink FM, de Jongh A, Aartman $\mathrm{IH}$ : What are people afraid of during dental treatment? Anxietyprovoking capacity of 67 stimuli characteristic of the dental setting. European journal of oral sciences 2008, 116(1):44-51.

16. Keogh E, Asmundson GJ: Negative affectivity, catastrophising and anxiety sensitivity. In: Understanding and treating fear of pain. edn. Edited by Asmundson GJ, Vlaeyen J, Crombez G. USA: Oxford University Press; 2004.

17. Lin CS, Wu SY, Wu LT: The anterior insula and anterior cingulate cortex are associated with avoidance of dental treatment based on prior experience of treatment in healthy adults. $B M C$ neuroscience 2015, 16:88.

18. Wang TF, Wu YT, Tseng CF, Chou C: Associations between dental anxiety and postoperative pain following extraction of horizontally impacted wisdom teeth: A prospective observational study. Medicine (Baltimore) 2017, 96(47):e8665.

19. Armfield JM: Development and psychometric evaluation of the Index of Dental Anxiety and Fear (IDAF-4C+). Psychol Assess 2010, 22(2):279-287.

20. McNeil DW, Helfer AJ, Weaver BD, Graves RW, Kyle BN, Davis AM: Memory of pain and anxiety associated with tooth extraction. Journal of dental research 2011, 90(2):220-224.

21. De Jongh A, Schutjes M, Aartman IH: A test of Berggren's model of dental fear and anxiety. European journal of oral sciences 2011, 119(5):361-365.

22. Olsson A, Phelps EA: Social learning of fear. Nature neuroscience 2007, 10(9):1095-1102.

23. Ogino $Y$, Nemoto H, Inui K, Saito S, Kakigi R, Goto F: Inner experience of pain: imagination of pain while viewing images showing painful events forms subjective pain representation in human brain. Cereb Cortex 2007, 17(5):1139-1146.

24. Moore R, Birn H: [Phenomenon of dental fear]. Tandlaegebladet 1990, 94(2):34-41.

25. Lin CS, Wu SY, Yi CA: Association between Anxiety and Pain in Dental Treatment: A Systematic Review and Meta-analysis. Journal of dental research 2017, 96(2):153-162.

26. Janz NK, Becker MH: The Health Belief Model: a decade later. Health Educ Q 1984, 11(1):1-47.

27. Cheng FC, Yu-Fong Chang J, Lin TC, Chang WC, Chang YT, Chiang CP: Dentist manpower development and geographical distribution of dentists in Taiwan. J Dent Sci 2020, 15(2):121-131.

28. Cheng TM: Taiwan's new national health insurance program: genesis and experience so far. Health Aff (Millwood) 2003, 22(3):61-76. 
29. Randall CL, McNeil DW, Shaffer JR, Crout RJ, Weyant RJ, Marazita ML: Fear of Pain Mediates the Association between MC1R Genotype and Dental Fear. Journal of dental research 2016, 95(10):1132-1137.

30. Yeung A, Goto TK, Leung WK: Brain responses to stimuli mimicking dental treatment among nonphobic individuals: A meta-analysis. Oral Dis 2019, 25(1):34-43.

\section{Tables}

Table 1 Results of the analysis of descriptive statistics across subjects 


\begin{tabular}{|c|c|c|c|c|c|c|c|c|}
\hline Total & Sex & Age & IDAF-4C+ & $\mathrm{HT}$ & ExpFear & ExplA & NExpFear & NExplA \\
\hline$n$ & 402 & 402 & 402 & 402 & 401 & 401 & 384 & 384 \\
\hline Male & 202 & & & & & & & \\
\hline Female & 200 & & & & & & & \\
\hline $\max$ & & 86 & 5.0 & $100 \%$ & 9.1 & 10.0 & 10.0 & 10.0 \\
\hline Q3 & & 60 & 2.1 & $83 \%$ & 5.0 & 2.8 & 6.3 & 4.0 \\
\hline median & & 47 & 1.4 & $67 \%$ & 3.1 & 1.2 & 4.3 & 1.8 \\
\hline Q1 & & 33 & 1.1 & $58 \%$ & 2.0 & 1.0 & 2.5 & 1.0 \\
\hline $\min$ & & 20 & 1.0 & $0 \%$ & 1.0 & 1.0 & 1.0 & 1.0 \\
\hline mean & & 47 & 1.8 & $67 \%$ & 3.6 & 2.2 & 4.5 & 2.8 \\
\hline S.D. & & 16 & 0.9 & $20 \%$ & 2.0 & 1.8 & 2.4 & 2.4 \\
\hline Community & Sex & Age & IDAF-4C+ & $\mathrm{HT}$ & ExpFear & ExplA & NExpFear & NExplA \\
\hline $\mathrm{n}$ & 201 & 201 & 201 & 201 & 200 & 200 & 188 & 188 \\
\hline Male & 105 & & & & & & & \\
\hline Female & 96 & & & & & & & \\
\hline $\max$ & & 86 & 5.0 & $100 \%$ & 8.9 & 10.0 & 10.0 & 10.0 \\
\hline Q3 & & 60 & 2.1 & $83 \%$ & 4.8 & 2.9 & 6.0 & 4.1 \\
\hline median & & 46 & 1.4 & $67 \%$ & 3.1 & 1.3 & 4.0 & 2.0 \\
\hline Q1 & & 30 & 1.1 & $58 \%$ & 2.0 & 1.0 & 2.3 & 1.0 \\
\hline $\min$ & & 20 & 1.0 & $0 \%$ & 1.0 & 1.0 & 1.0 & 1.0 \\
\hline mean & & 45 & 1.8 & $65 \%$ & 3.6 & 2.3 & 4.4 & 2.9 \\
\hline S.D. & & 17 & 0.9 & $21 \%$ & 2.0 & 1.9 & 2.4 & 2.4 \\
\hline Clinical & Sex & Age & IDAF-4C+ & $\mathrm{HT}$ & ExpFear & ExplA & NExpFear & NExplA \\
\hline$n$ & 201 & 201 & 201 & 201 & 201 & 201 & 196 & 196 \\
\hline Male & 97 & & & & & & & \\
\hline Female & 104 & & & & & & & \\
\hline $\max$ & & 86 & 5.0 & $100 \%$ & 9.1 & 9.1 & 10.0 & 10.0 \\
\hline Q3 & & 61 & 2.0 & $83 \%$ & 5.0 & 2.5 & 6.5 & 4.0 \\
\hline median & & 48 & 1.4 & $67 \%$ & 3.1 & 1.1 & 4.5 & 1.6 \\
\hline
\end{tabular}




\begin{tabular}{|c|c|c|c|c|c|c|c|c|}
\hline Q1 & & 37 & 1.0 & $58 \%$ & 2.0 & 1.0 & 2.7 & 1.0 \\
\hline $\min$ & & 20 & 1.0 & $8 \%$ & 1.0 & 1.0 & 1.0 & 1.0 \\
\hline mean & & 49 & 1.7 & $68 \%$ & 3.6 & 2.1 & 4.7 & 2.7 \\
\hline S.D. & & 16 & 0.9 & $18 \%$ & 2.1 & 1.7 & 2.4 & 2.3 \\
\hline Comparison ${ }^{*}$ & n.s. & n.s. & n.s. & n.s. & n.s. & n.s. & n.s. & n.s. \\
\hline
\end{tabular}

*Due to the non-normality of the distribution of all the variables, comparison between two subgroups was performed using Mann-Whitney U test.

ExpFear: fear of experienced conditions, ExplA: intention of avoidance of experienced conditions, $\mathrm{HT}$ : history of treatment, IDAF-4C+: the Index of Dental Anxiety and Fear, NExpFear: fear of non-experienced conditions, NExpIA: intention of avoidance of non-experienced conditions, n.s.: statistically not significant, Q1: the first quartile, Q3: the third quartile, S.D.: standard deviation.

Table 2 Results of the analysis of descriptive statistics of each procedure 


\begin{tabular}{|c|c|c|c|c|c|c|c|}
\hline \multirow[t]{2}{*}{ Conditions of dental treatment } & \multirow[t]{2}{*}{ Prevalence } & \multicolumn{2}{|c|}{ Fear } & \multicolumn{2}{|l|}{$\mathrm{IA}$} & \multicolumn{2}{|c|}{$\begin{array}{l}\text { Magnification } \\
\text { factor }\end{array}$} \\
\hline & & Exp & NExp & Exp & NExp & Fear & IA \\
\hline $\begin{array}{l}\text { Receiving a local anesthetic injection } \\
\text { in the mouth }\end{array}$ & $94 \%$ & 4.2 & 5.4 & 2.1 & 3.9 & $30 \%$ & $84 \%$ \\
\hline $\begin{array}{l}\text { Having a painful tooth tapped by the } \\
\text { dentist }\end{array}$ & $85 \%$ & 3.7 & 4.3 & 2.2 & 2.7 & $14 \%$ & $23 \%$ \\
\hline $\begin{array}{l}\text { Having a primary tooth (milk teeth) } \\
\text { extracted in the childhood }\end{array}$ & $48 \%$ & 4.5 & 4.3 & 3.2 & 3.3 & $-3 \%$ & $2 \%$ \\
\hline $\begin{array}{l}\text { Receiving ultrasonic scaling for } \\
\text { removing dental stone }\end{array}$ & $92 \%$ & 2.5 & 2.5 & 1.7 & 2.1 & $-1 \%$ & $21 \%$ \\
\hline A molar being drilled to remove caries & $85 \%$ & 4.0 & 3.9 & 2.4 & 2.5 & $-4 \%$ & $6 \%$ \\
\hline Receiving a root canal treatment & $68 \%$ & 4.6 & 6.1 & 2.6 & 4.0 & $33 \%$ & $56 \%$ \\
\hline $\begin{array}{l}\text { Having a wisdom tooth extracted by } \\
\text { surgery }\end{array}$ & $38 \%$ & 4.8 & 5.9 & 2.7 & 3.7 & $22 \%$ & $37 \%$ \\
\hline $\begin{array}{l}\text { Feeling painful hypersensitivity when } \\
\text { rinsing cold water }\end{array}$ & $77 \%$ & 2.5 & 2.0 & 1.7 & 1.6 & $-22 \%$ & $-5 \%$ \\
\hline $\begin{array}{l}\text { A caries tooth being explored with a } \\
\text { dental instrument }\end{array}$ & $91 \%$ & 3.8 & 3.9 & 2.2 & 2.5 & $3 \%$ & $13 \%$ \\
\hline $\begin{array}{l}\text { Having the swelling gum incised and } \\
\text { pus drained }\end{array}$ & $33 \%$ & 4.1 & 5.0 & 2.4 & 2.9 & $22 \%$ & $18 \%$ \\
\hline $\begin{array}{l}\text { Feeling excruciating postoperative } \\
\text { pain; not being relieved even with } \\
\text { painkillers }\end{array}$ & $34 \%$ & 4.5 & 4.1 & 2.8 & 2.4 & $-9 \%$ & $-14 \%$ \\
\hline $\begin{array}{l}\text { Receiving a wedge and band in } \\
\text { between the teeth during restoration }\end{array}$ & $54 \%$ & 2.6 & 3.5 & 1.9 & 2.4 & $35 \%$ & $27 \%$ \\
\hline
\end{tabular}

Exp: experienced conditions; IA: intention of avoidance, NExp: non-experienced conditions.

\section{Figures}


(A)

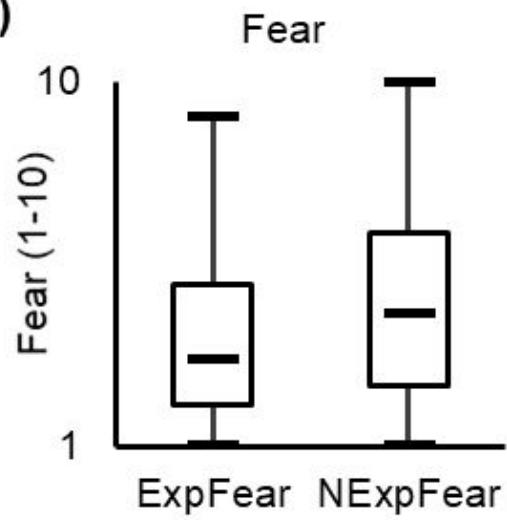

(B)

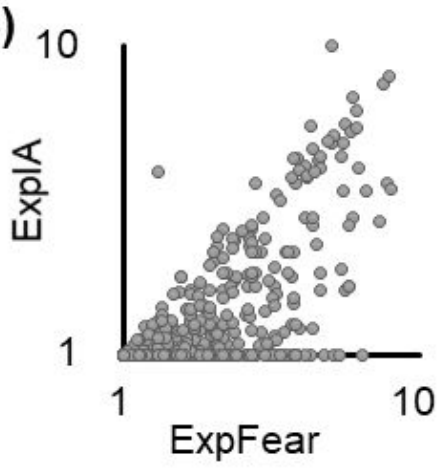

(C)

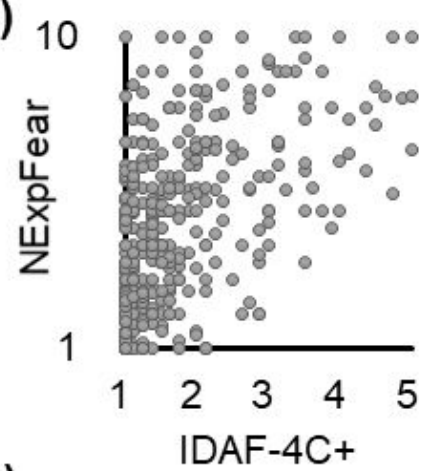

(D)

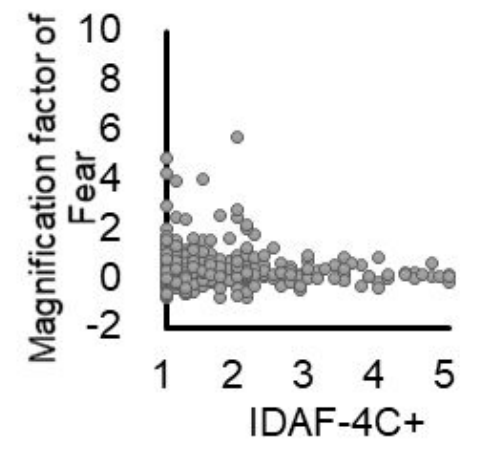

Intention of avoidance (IA)
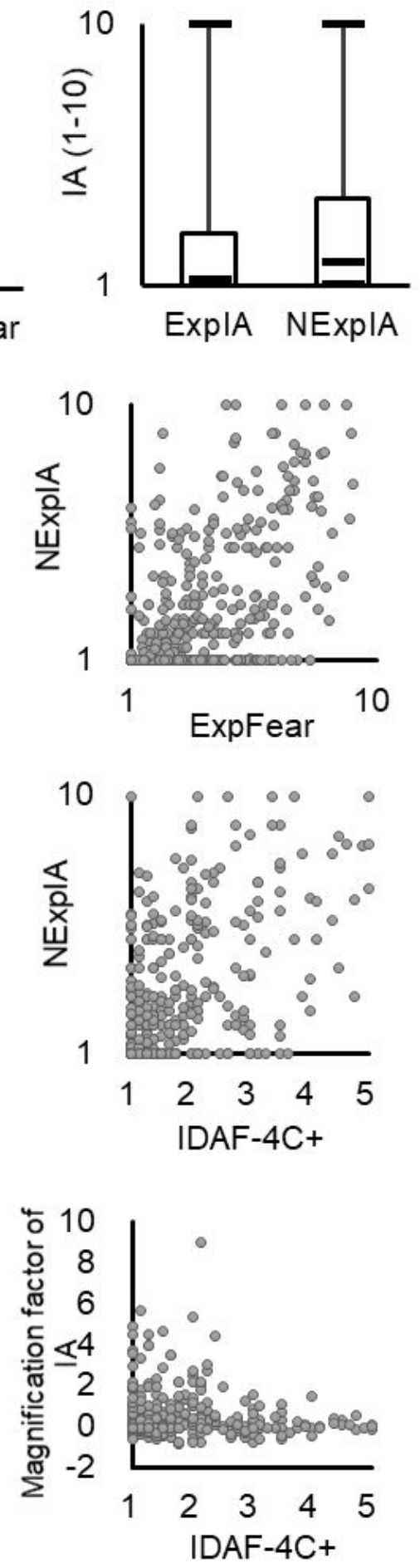

\section{Figure 1}

Association between experienced and non-experienced fear/intention of avoidance (IA). (A) Fear of nonexperienced conditions (NExpFear) is significantly higher than fear of experienced conditions (ExpFear). IA of non-experienced conditions (NExpIA) is significantly higher than fear of experienced conditions (ExpIA). (B) ExpFear is significantly positively correlated with ExpIA and IA of non-experienced conditions (NExpIA). (C) Trait dental anxiety (IDAF-4C+ score) is significantly positively correlated with fear of non- 
experienced conditions (NExpFear) and NExpIA. (D) Trait dental anxiety is significantly negatively correlated with the magnification factor of fear but not that of IA.

(A)

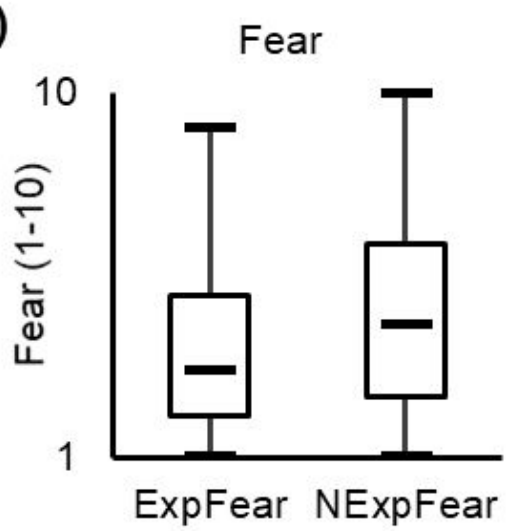

(B)

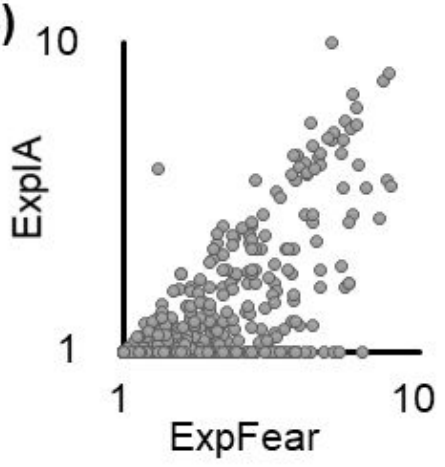

(C)

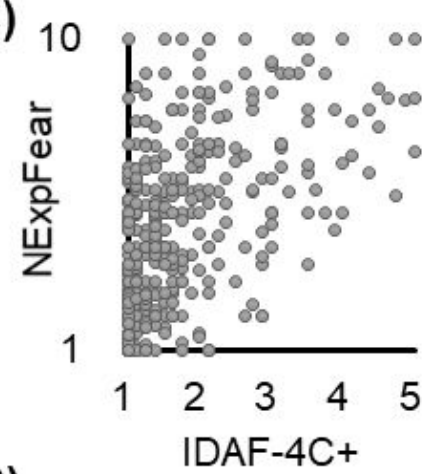

(D)

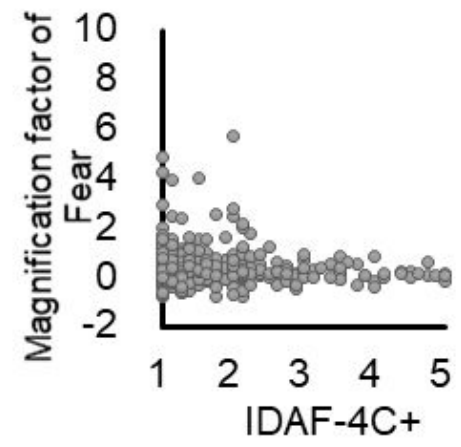

Intention of avoidance $(I A)$
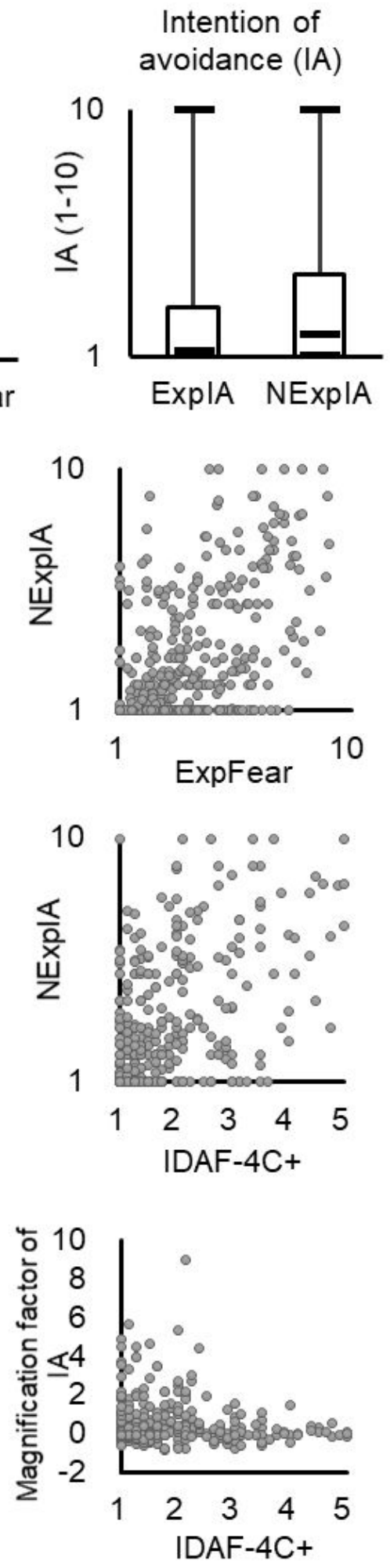

Figure 1

Association between experienced and non-experienced fear/intention of avoidance (IA). (A) Fear of nonexperienced conditions (NExpFear) is significantly higher than fear of experienced conditions (ExpFear). IA of non-experienced conditions (NExpIA) is significantly higher than fear of experienced conditions 
(ExpIA). (B) ExpFear is significantly positively correlated with ExpIA and IA of non-experienced conditions (NExpIA). (C) Trait dental anxiety (IDAF-4C+ score) is significantly positively correlated with fear of nonexperienced conditions (NExpFear) and NExpIA. (D) Trait dental anxiety is significantly negatively correlated with the magnification factor of fear but not that of IA.

\section{(A)}

\section{Fear by conditions}

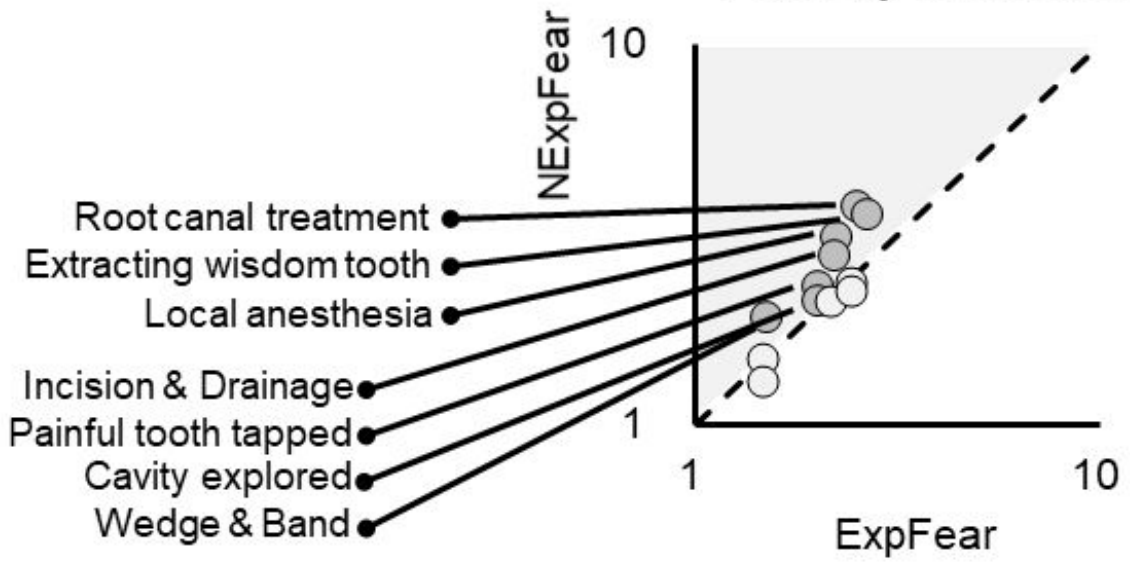

IA by conditions

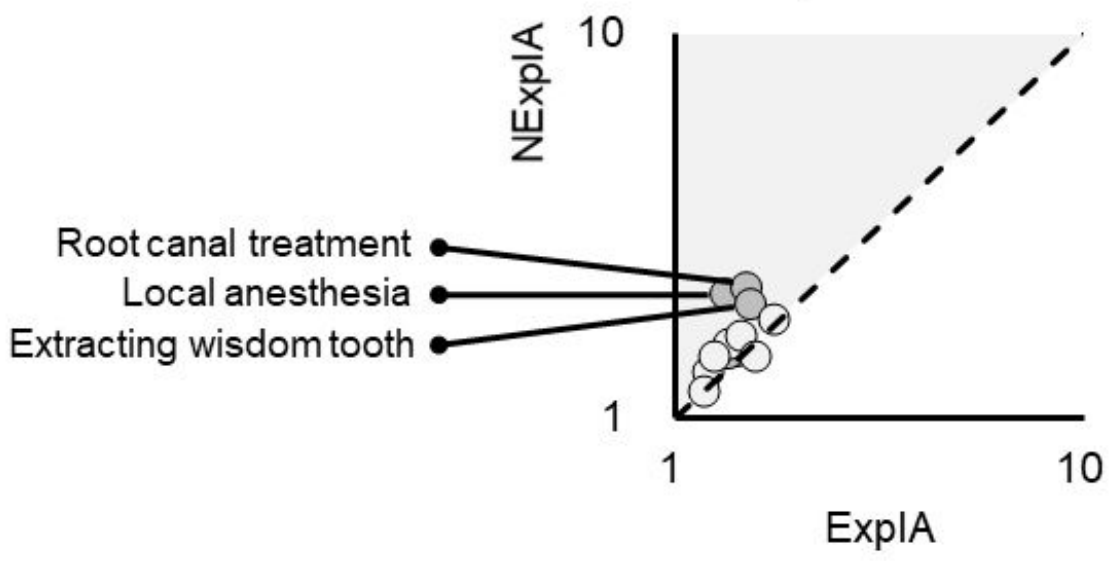

(B)
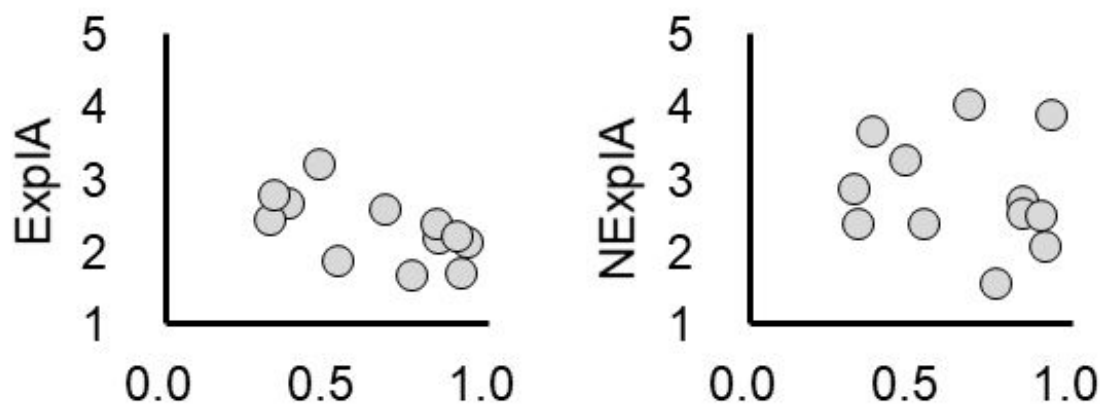

Prevalence

Prevalence

Figure 2 
Analysis of fear and intention of avoidance (IA) by conditions of dental treatment. (A) Fear of subjects have an experience (ExpFear) and subjects without an experience (NExpFear) is significantly positively correlated across the 12 conditions. IA of subjects have an experience (ExpIA) and subjects without an experience (NExpIA) is not significantly correlated. The area with a gray shade indicates the conditions with a magnification of fear/IA in non-experienced subjects. (B) The prevalence of experiencing a condition is significantly negatively correlated with ExpIA but not NExpIA.
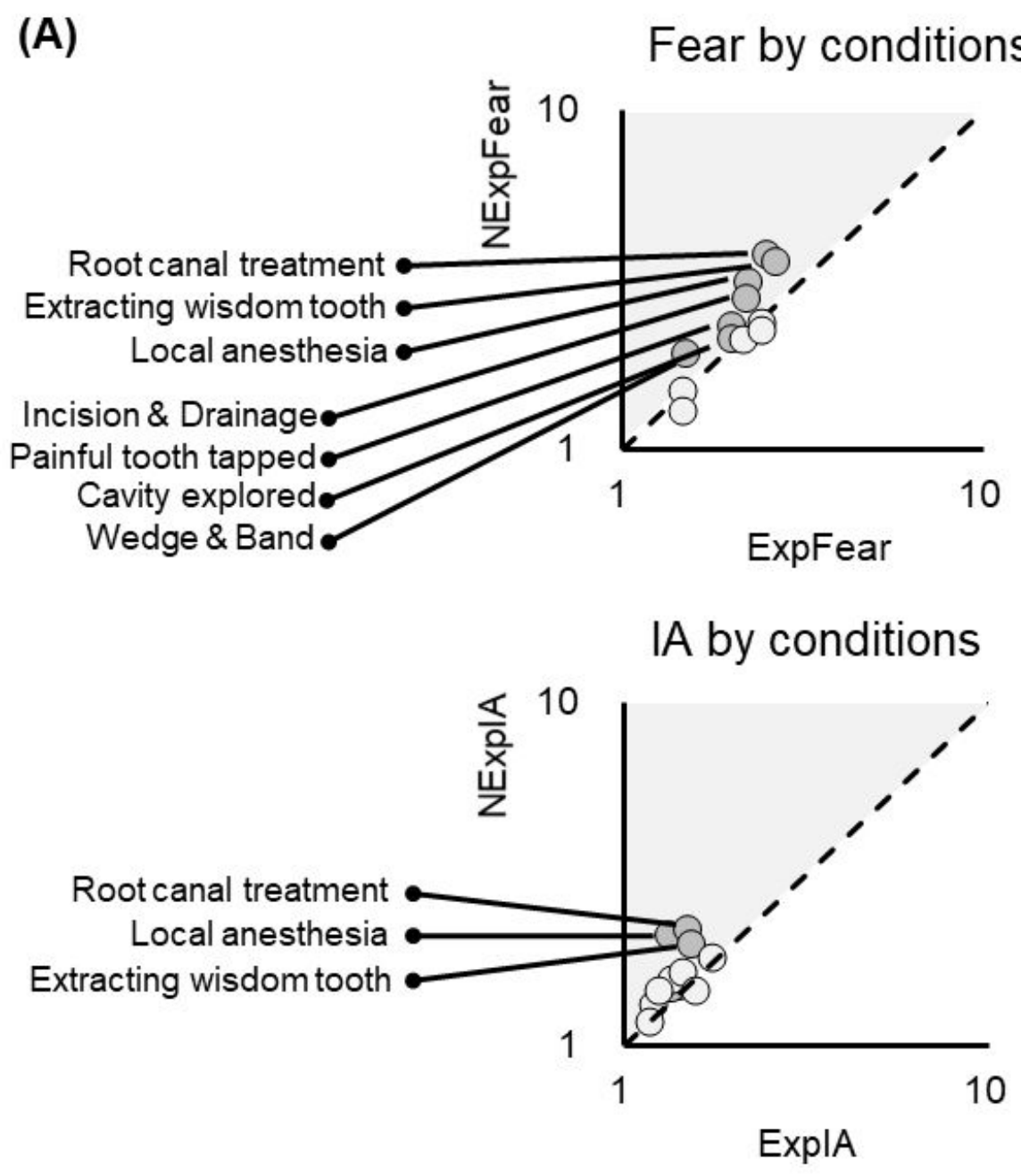

(B)
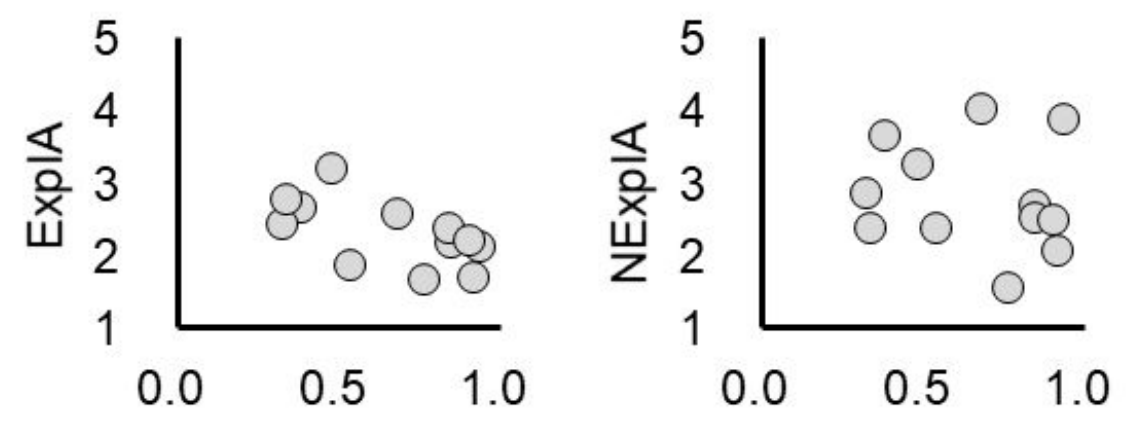

Prevalence

Prevalence

Figure 2 
Analysis of fear and intention of avoidance (IA) by conditions of dental treatment. (A) Fear of subjects have an experience (ExpFear) and subjects without an experience (NExpFear) is significantly positively correlated across the 12 conditions. IA of subjects have an experience (ExpIA) and subjects without an experience (NExpIA) is not significantly correlated. The area with a gray shade indicates the conditions with a magnification of fear/IA in non-experienced subjects. (B) The prevalence of experiencing a condition is significantly negatively correlated with ExpIA but not NExpIA. 